This is the final peer-reviewed accepted manuscript of:

M. Dini, A. Romani, M. Filippi and M. Tartagni

A Nanopower Synchronous Charge Extractor IC for Low-Voltage Piezoelectric Energy Harvesting With Residual Charge Inversion

in IEEE Transactions on Power Electronics, vol. 31, no. 2, pp. 1263-1274, Feb. 2016

The final published version is available online at:

https://doi.org/10.1109/TPEL.2015.2417352

Rights / License:

The terms and conditions for the reuse of this version of the manuscript are specified in the publishing policy. For all terms of use and more information see the publisher's website.

This item was downloaded from IRIS Università di Bologna (https://cris.unibo.it/)

When citing, please refer to the published version. 


\title{
A Nano-Power Synchronous Charge Extractor IC for Low Voltage Piezoelectric Energy Harvesting with Residual Charge Inversion
}

\author{
Michele Dini, Aldo Romani, Matteo Filippi, and Marco Tartagni, Member, IEEE
}

\begin{abstract}
The paper presents a power converter for piezoelectric energy harvesting implementing a modified version of synchronous electrical charge extraction with quiescent current as low as $160 \mathrm{nA}$. The input energy is increased of more than $200 \%$ for weak vibrations, by inverting the residual charge left on the capacitance of the transducer after each energy extraction. Moreover, a power management policy, named twoway energy storage, is introduced in order to improve significantly the efficiency of the energy harvesting system in battery-less systems during the start-up phase, when the energy storage is fully depleted. The converter behaves as a buck-boost converter and the measured peak efficiency is $85.3 \%$. The IC has been designed in a $0.32 \mu \mathrm{m}$ microelectronic technology from STMicroelectronics in an active area of $0.95 \mathbf{~ m m}^{2}$.
\end{abstract}

Index Terms-piezoelectric transducers, energy harvesting, power converter, power management, nano-power.

\section{INTRODUCTION}

$\mathrm{T}$ HE field of piezoelectric energy harvesting has been undergoing continuous advances over the last years. In this context, systems make use of piezoelectric transducers and of dedicated electronic interfaces in order to extract power from environmental energy sources such as vibrations, shocks and human movement with the purpose of ubiquitously supplying miniaturized autonomous electronic systems, e.g. wearable or environmental sensors and wireless nodes. The relative high energy density and relative ease of fabrication of piezoelectric materials [1] make them promising candidates for pursuing this goal.

Several types and shapes of piezoelectric transducers have been reported in literature targeting many different types of applications [2][3]. More specifically, a growing effort is devoted to the miniaturization of transducers, key for unobtrusive applications [4]. This means that energy harvesting systems and applications have to deal with very limited power levels, since optimized electro-mechanical

This research has received funding from the European Community under the ENIAC Nanoelectronics Framework Programme (Call ENIAC-2012-2, grant agreement Lab4MEMS no.325622-2) and from the Italian Ministry of Instruction, Research and University (MIUR), within the framework of the national project PRIN 2011 GRETA.

M. Dini, A. Romani, M. Filippi and M. Tartagni are with the Department of Electrical, Electronic, and Information Engineering "Guglielmo Marconi“" of the University of Bologna, Cesena 47521, Italy, (e-mail: michele.dini3@unibo.it, aldo.romani@unibo.it, matteo.filippi6@unibo.it, marco.tartagni@unibo.it; phone: +39 0547 339537; fax: +39 0547 339208). designs with macro-scale transducers yield power densities as low as $10-100 \mu \mathrm{W} / \mathrm{cm}^{3}$ in many practical cases [5]. In addition, the current trend is to further shrink down transducers with MEMS fabrication processes [6][7], with available power levels down to few $\mu \mathrm{W}$.

For this reason, a special care has to be put in designing efficient electrical interfaces for power conversion and energy storage with very low intrinsic consumption and power losses. Since the beginning, interfaces based on passive diode rectifiers [8] or switching step-down converters with fixed or variable duty-cycles [9][10] have been proposed for transferring electrical charge from a piezoelectric transducer to a storage capacitor. However, diode voltage drops significantly limit the harvested power especially in case of low input voltages, as it is the case in many energy harvesting applications. Moreover, no power is harvested when the input voltage is lower than that on the output node, introducing an intrinsic limitation to the storable energy.

Such types of limitations were in part overcome by synchronized switch harvesters [11], a category of power converters based on resonant circuits activated in a way synchronous with vibrations. Thanks to the mainly capacitive impedance of piezoelectric transducers (Fig. 1), such interfaces increase both the peak voltage and the harvested power. A notable example is the SSHI technique [13], in which the transducer is connected to a rectifier bridge and to a (a)

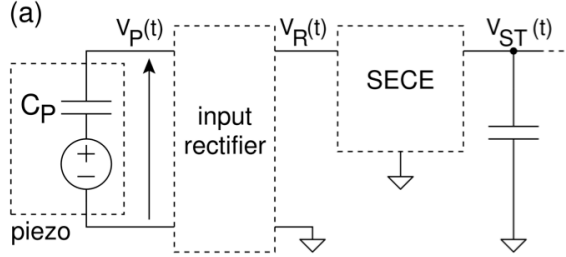

(b) boost SECE

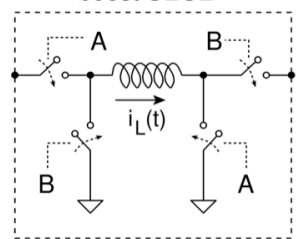

(c) flyback SECE

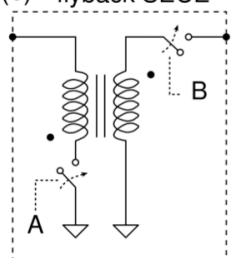

(d)

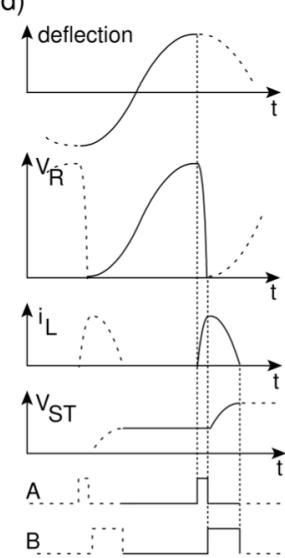

Fig. 1. (a) Structure of a power converter stage based on synchronous electrical charge extraction (SECE) for piezoelectric transducers. (b) a boost topology for SECE. (c) a flyback topology for SECE. (d) Representative waveforms of SECE under the assumption of ideal rectification 
parallel connected switched inductor used for inverting its voltage at the maximum elongation points, i.e. when the bare passive rectifier stops conducting. Such inversion brings the rectifier near the opposite conduction threshold and improves conduction during the next elongation. However, electrical charge is still transferred through the rectifier, making efficiency bias dependent and quite low in case of very low or very high load currents. Another known technique is synchronous electrical charge extraction (SECE) [12][13] in which, when a voltage peak is detected, energy is first transferred from the transducer to an inductor and then to the output through two resonant circuits, in a way similar to a boost converter, as shown in Fig. 1. SECE can also be implemented with a flyback topology [14]-[16] in order to reduce losses on freewheeling diodes. However, the resulting longer switching transients may yield higher energy losses, especially in case of large capacitive loads. Conveniently, the efficiency of SECE does not depend on the output bias, while the complete removal of electrical charge at each activation doubles the peak voltage. Besides this, SSHI [17] and more advanced synchronized harvesters based on multi stage converters or transformers, such as DSSH [18] or SSHI-MR [19] have also been reported to increase the harvested power. On the other hand, such techniques are strongly related to the load condition (SSHI-MR) whereas SECE is almost loadindependent or are based on a dual-stage architecture which requires two inductors and is likely addressed to consume more power due to increased control and switching activity.

One of the main drawbacks of the above mentioned interfaces is the use of diodes in practical circuit implementations, as in [13][20][21], whose voltage drops significantly limit the harvested power especially in case of low input voltages, as it is the case in many energy harvesting applications. Alternative solutions, such as cross coupled MOSFETs rectifiers, i.e. negative voltage converters (NVCs) [22], active rectifiers [22] or digitally controlled switches [23] have been demonstrated to improve the power performance. NVCs offer the potential of zero-threshold rectification but allow bidirectional current flows and also suffer from bad performance in case of low input voltages, i.e. lower than the threshold voltage of MOSFETs. Active rectifiers reduce conduction losses at the expense of additional power consumption and are currently promising solutions mainly for integrated circuit implementations where bias currents can be strongly reduced [24][25].

In energy harvesting applications, since replacing or recharging batteries is often problematic, another important aspect is the possibility of designing self-powered battery-less circuits, such for example the SSHI interface in [20] and the SECE circuit in [26], consuming only few $\mu$ A. Electrical charge is usually stored on low-leakage capacitors sized according to the actual load requirements. In many targeted applications of wireless sensor networks this constraint requires the use of supercapacitors [27], fostered by significant advances towards the reduction of geometries [28] and leakage currents [29]. Despite this, the use of large capacitances in resonant power converters such as the previously mentioned ones, combined with the further constraint of designing micro-power control circuits with limited operating frequencies and bandwidths, may lead to low electrical quality factors, as it will be pointed out later on.

Recently, several integrated circuit for energy harvesting have been proposed [30]-[33] with power consumption in the order of hundreds of $\mathrm{nW}$. Silicon implementation of converters allows, besides a smaller footprint and more complex fully customizable architectures, a reduction of power of at least an order of magnitude with respect to optimized discrete components realizations [34]. Indeed, specific energy aware circuital design techniques, converter topology and silicon implementation allow the exploitation of ultra-low power sources with a positive output energy budget. The use of such sources, and of the associated power levels, would be prevented by a design using discrete components and the harvesting effectiveness is likely to be compromised.

This paper will introduce a series of circuit techniques for optimizing charge collection and power management in piezoelectric energy harvesters subject to weak vibrations. As a first contribution, the residual charge on transducers will be exploited for achieving improved energy conversion efficiency, with respect to existing solutions, when operating with low voltages. Besides this, the paper will introduce a power management policy for selectively routing the harvester power to the application load or for internal needs, with a resulting faster start-up time and higher efficiency than conventional solutions. Such techniques will be considered in the perspective of nano-power integrated circuit design, tackling realistic application constraints, including switch implementation, minimization of intrinsic power consumption and full energy autonomy. The design will be validated through the characterization of prototypes based on a full custom silicon design.

\section{SECE WITH RESIDUAL CHARGE INVERSION}

A careful design of the input interface is mandatory in order to achieve high efficiencies while extracting charge from piezoelectric transducers, especially when low voltages are involved. The more efficient this process is, the higher the duty cycle of operation of the final application, e.g. data acquisition and wireless transmissions, will be. In energy harvesting systems, energy should be collected as long as it is available from the environmental sources. For this reason, a buck-boost topology is a suitable candidate as input stage. In fact, in passive rectifiers and step-down converters the output voltage cannot exceed that on the input whereas it is likely required to extract energy in the opposite situation. Among buck-boost topologies, piezoelectric transducers subject to weak and irregular vibrations are efficiently handled with SECE, which is also compatible with micro-power control circuits [26]. In self-powered implementations of SECE, since piezoelectric voltages often switch from negative to positive voltages, the difficulty of generating dual voltage supplies is usually overcome by using input rectifier stages, whose 

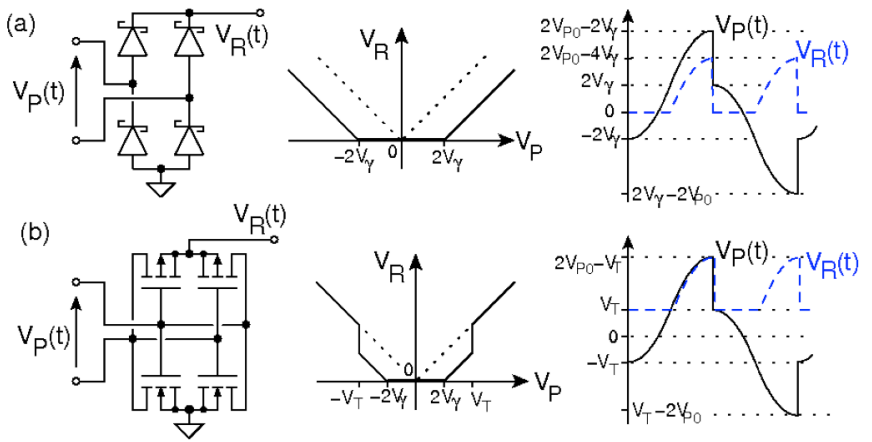

Fig. 2. Schematic, voltage transfer characteristic and transient behavior during SECE with: (a) BR; (b) NVC.

voltage drops, however, limit efficiency in case of low input voltages. Energy conversion, as mentioned in Section I and shown in Fig. 1, is activated on local maxima of the rectified voltage. Energy is first extracted from the piezoelectric capacitance $C_{P}$ with a switched inductor $L_{l}$ forming a $L_{l}-C_{P}$ resonant circuit. Then, energy is transferred from $L_{l}$ into a storage capacitor $C_{O}$ by forming a second $L_{I}-C_{O}$ resonant circuit. Since the $L_{1}-C_{O}$ resonant circuit is never connected to the piezoelectric transducer, a measure of the capability of a SECE interface to extract power is given by the energy stored in the inductor at the end of the first phase.

Let us suppose that $V_{P}(t)=V_{P 0} \sin (2 \pi f t)$ is the voltage generated across the transducer in open circuit, where $f$ is the vibration frequency. If a full-wave bridge rectifier (BR) is adopted (Fig. 2a) as input interface, in case of conduction the rectified voltage is $V_{R}(t)=\left|V_{P}(t)\right|-2 V_{r}$, where $V_{y}$ is the threshold voltage of a single diode and $V_{R}(t) \geq 0$. When SECE is activated on a voltage peak, the transducer is discharged through the rectifier and the inductor until $V_{R}(t)=0$. Then, the rectifier turns off and a residual voltage $\pm 2 V_{\text {, }}$ is left on $V_{P}(t)$. From this condition, since a peak-to-peak elongation produces a voltage variation $2 V_{P 0}$ on the transducer, a maximum voltage $2\left(V_{P 0}-V_{v}\right)$ can be reached on $V_{P}(t)$.

If we define $\gamma=V / V_{P 0}$, with $0<\gamma<0.5$, the energy stored in $L_{l}$ at the end of the first phase can be computed by solving the differential equations of the $L_{l}-C_{P}$ circuit, as demonstrated in [34]:

$E_{L}^{(B R)}=2 C_{P} V_{P 0}^{2}(1-2 \gamma)^{2} e^{-\pi /\left(\omega_{01} \tau\right)}$,

where $\tau=2 L_{l} / R_{l}$, with $R_{l}$ assumed to be the resistance of switches, inductor and transducer of $L-C_{P}$, and $\omega_{01} \cong \sqrt[-1]{L_{1} C_{P}}$.

In case a NVC is used (Fig. 2b), $V_{R}(\mathrm{t})=V_{P}(\mathrm{t})$ as long as $\left|V_{P}(\mathrm{t})\right|>V_{r}$, where $V_{r}$ is the absolute value of the highest MOSFET threshold voltage. With respect to diodes, MOSFETs offer negligible voltage drops and energy losses. However, on the activation of SECE, during the discharge of $V_{P}(t)$, the NVC turns off when $\left|V_{P}(t)\right|=V_{r}$. For lower voltages, conduction may still occur through the FET body diodes, which would introduce significant losses, so that energy extraction should safely stop at $V_{T}$. Then, at the end of the subsequent elongation, a maximum absolute voltage $2 V_{P 0}-V_{r}$ will be reached on $V_{P}(t)$.
If we define $\delta=V_{T} / V_{P 0}$, with $0<\delta<1$, the energy stored in $L_{l}$ after the transducer has discharged from $2 V_{P 0}-V_{r}$ to $V_{T}$ can be determined:

$E_{L}^{(N V C)}=2 C_{P} V_{P 0}^{2}(1-\delta) e^{\frac{-2 \arccos \left(\frac{\delta}{2-\delta}\right)}{\omega_{01} \tau}}$.

In the above two cases no power is harvested for absolute input voltages lower than the minimum conduction thresholds of $2 V_{r}$ and $V_{T}$, and residual charges $\left|Q_{B R}\right|=2 C_{P} V_{r}$ and $\left|Q_{N V C}\right|=C_{P} V_{T}$ are left at the end of every conversion. Such residual charge has to be first canceled during the subsequent peak-to-peak elongation before the sign of $V_{P}(t)$ changes.

As a term of comparison, a lossless SECE with an ideal rectifier with $V_{r}=0$ (IR) would leave no residual charge and store in $L_{l}$ the following energy for a single activation:

$E_{L}^{(I R)}=2 C_{P} V_{P 0}^{2} e^{-\pi /\left(\omega_{01} \tau\right)}$

In this section we propose an input interface based on a NVC with residual charge inversion (RCI), shown in Fig. 3. This allows to reduce energy losses through the MOSFET bridge and to remove all the charge $Q_{P P}=2 C_{P} V_{P 0}$ generated in a peak-to-peak elongation. The inversion of residual charge applies a more favorable voltage offset for the next peak-topeak elongation. Other types of pre-biasing techniques, in which the offset charge is drawn from the output, were introduced in [35] and showed to significantly increase the performance. However, in case of low output voltages, e.g. when high load currents are applied, the advantages of the prebias are reduced. Differently, this work exploits as a bias the inverted residual charge, which otherwise would impact negatively output power. This approach improves the

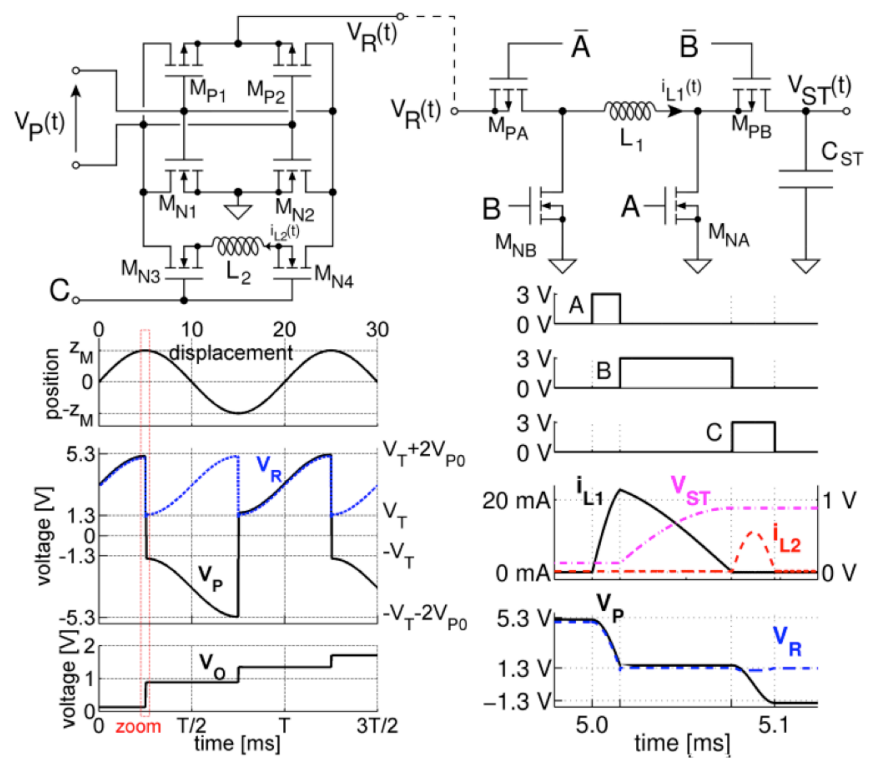

Fig. 3. Schematic and circuit simulations of a SECE circuit based on a NVC with the proposed RCI. Circuit simulations were performed with $V_{P 0}=2 \mathrm{~V}$, $f=50 \mathrm{~Hz}, L_{1}=10 \mathrm{mH}, L_{2}=2.5 \mathrm{mH}, C_{O}=1 \mu \mathrm{F} . \mathrm{M}_{\mathrm{N} 1 . .4}$ and $\mathrm{M}_{\mathrm{P} 1,2}$ are standard discrete MOSFETs with $\left|V_{T}\right|=1.3 \mathrm{~V}$ A zoomed view of an individual energy conversion is also shown. 
performance especially in case of low vibrations, when the input voltage is comparable to the conduction threshold and the losses would otherwise be significant. The inversion of residual charge is also performed in other synchronized switch techniques, such as for example SSHI, in which charge inversion is mostly required for keeping a rectifier bridge in a conducting state for most of the time. However, the conversion efficiency is still bias dependent. Differently, besides producing significantly higher piezoelectric voltages, performing RCI with SECE also introduces a bias independent energy conversion efficiency, because the transducer is never directly connected to the output node.

As shown in Fig. 3, with respect to SECE, an inductor $L_{2}$ and two switches $M_{N 3}, M_{N 4}$ are introduced. An additional signal $\mathrm{C}$ is activated for inverting the residual charge left on the transducer. This is accomplished by letting the resonant circuit $C_{P}-L_{2}$ oscillate for a half period $\pi / \omega_{02}$, where $\omega_{02} \cong 1 / \sqrt{L_{2} C_{P}}$ is its resonance frequency. This new initial offset would ideally allow to reach a higher maximum voltage $2 V_{P 0}+k V_{T}$ on $V_{P}(t)$, where $k=\exp \left(-\pi /\left(\omega_{02} \tau_{2}\right)\right), \tau_{2}=2 L_{2} / R_{2 e q}$ and $R_{2 e q}$ is the series resistance of $L_{2}$ and of the RCI switches ( $\mathrm{M}_{\mathrm{N} 3}$ and $\mathrm{M}_{\mathrm{N} 4}$ in Fig. 3). It can be found that the energy stored in $L_{I}$ after the transducer has discharged from $2 V_{P 0}+k V_{T}$ to $V_{T}$ is:

$$
E_{L}^{(R C I)}=2 C_{P} V_{P 0}^{2}\left(1+k \delta+\frac{k^{2}-1}{4} \delta^{2}\right) e^{\frac{-2 \arccos \left(\frac{\delta}{2+k \delta}\right)}{\omega_{01} \tau}}
$$

Typical values for the components that have been considered for analytical evaluations are $V_{\gamma} \cong 0.35 \mathrm{~V}$ for low threshold Schottky diodes, e.g. BAT754, and $V_{T} \cong 1.3 \mathrm{~V}$ for discrete MOSFETs with low gate charge and compatible with piezoelectric voltages of up to $20 \mathrm{~V}$, e.g. BSS138PW and NTR1P02T1, and $V_{T} \cong 1 \mathrm{~V}$ for generic integrated MOSFETs. Hence, in practical cases, it roughly holds that $\delta / \rho \cong 2 \ldots 3$.

The corresponding energy ratios of (1), (2) and (4) normalized to $2 C_{P} V_{P 0}{ }^{2}$, i.e. $E_{0}$, are functions of $\delta$ and $\rho$ for a given set of circuit parameters. A comparison plot is shown in Fig. 4. As it can be observed, Schottky rectifiers underperform with respect to $\mathrm{NVC}$ and $\mathrm{RCI}$ is the best option, especially for

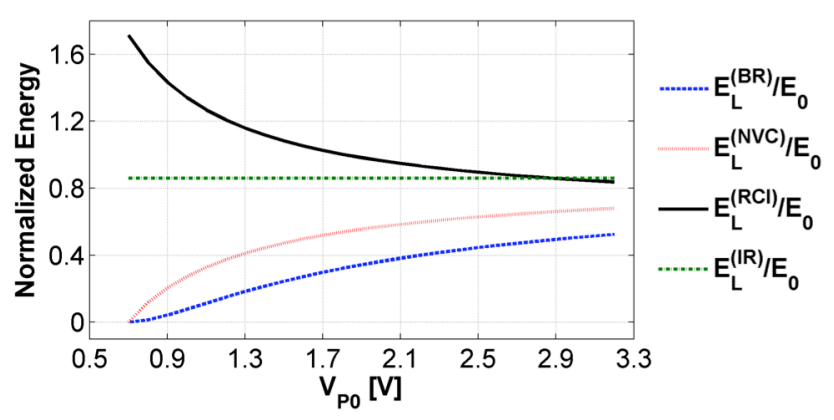

Fig. 4. Normalized energy $\left(E_{0}=2 C_{P} V_{P 0}{ }^{2}\right)$ extracted from $C_{P}$ and stored in $L_{I}$ at the end of the first phase of SECE with different types of the input interface: full-wave bridge rectifier (BR), NVC, NVC with RCI enabled (RCI), and ideal rectifier (IR). The parameters used for the generation of the figure are: $V_{T} \cong 1 \mathrm{~V}, V_{\gamma}=0.35 \mathrm{~V}, C_{P}=52 \mathrm{nF}, L_{1}=L_{2}=560 \mu \mathrm{H}, R_{l}=10 \Omega, R_{2 e q}=5 \Omega$. low input voltages. However, this holds for an integrated circuit perspective. For a discrete components design, Schottky rectifiers perform better than NVC for low input voltage due to typical high MOSFET $\mathrm{V}_{\mathrm{T}}$ unless RCI is employed.

The OSECE topology [15] is an interesting improvement of classic SECE topology. Differently from the latter, it exploits three coupled inductors and diodes. OSECE has a lower circuit complexity (switches and their controllers) and is surely more suitable than SECE for an implementation with off-the-shelf components, although some works implementing SECE converters have been reported [34]. However, in order to improve efficiency, reduce size and costs on large volumes, an integrated solution is advisable. Several works [30][33][36] use active rectifiers for diodes replacement as they have lower losses and lower inverse leakage current than diodes. The command energy required for the MOSFET and its driver is very small for integrated circuits compared to PCB circuits with discrete components and, furthermore, it can be tailored on application requirements (i. e. switching frequency, turn-on delay and on-resistance). In such a perspective, an integrated version of OSECE with active rectifiers might offer better performance than the PCB solution and even outperform an integrated SECE. A drawback of active switches and rectifiers is their inability to operate without a supply and therefore a start-up mechanism is required (e.g. a secondary passive rectifier in parallel with the active one).

However, a significant difference between SECE with RCI and OSECE lie in the amount of inverted charge on the piezoelectric transducer. With SECE-RCI such amount of charge is independent from both the load and the input parameters and depends only on the characteristics of the MOSFETs employed in the NVC (i.e. their $V_{G S, t h}$ ). Differently, in OSECE the amount of inverted charge strongly depends on the output voltage (i.e. load), transformer turns ratio and diodes characteristics. With high turns-ratio of the transformer, the inverted charge might be very low and provide a less favorable offset than SECE-RCI.

\section{Two-WAy ENERGy StORAGE AND Power MANAGEMENT POLICY}

A typical issue of self-powered harvesting systems exploiting active converters (i.e. not a bare BR) is the start-up time required for transition from passive to active harvesting mode. Systems with a single energy storage element $[21,22,28-30]$ rely on such energy reservoir both for supplying both the converter supply and the load. Since application requirements, e.g. for sustaining a wireless sensor node transmission, require a minimum amount of stored energy and a minimum voltage to enable operation, usually large capacitors or supercapacitors are used. As a consequence, a considerable amount of time ranging from seconds to hours [30][37] may be required for switching from a passive harvesting interface to an active power conversion interface, which also require a minimum operating voltage. During this period, the efficiency of energy extraction is negatively affected. A second issue is the inability of the load to consume 
all the energy in the storage element without compromising the operations of the converter by bringing it back into passive mode. This limits the energy available to the load because a considerable amount of energy is locked in the storage capacitor just for keeping the output voltage high enough for enabling the power converter and without the possibility of being used by the load. In systems with a huge energy storage, e.g. a tens of $\mathrm{mF}$ supercapacitor, such amount of wasted energy is intolerable, especially if a second buck-boost regulator is placed between the harvester and the load with the task of generating a stable and regulated supply voltage

The proposed power management policy is similar to that introduced in [16], [38]-[40] and makes use of two different capacitors, as shown in Fig. 5: the converter power supply is provided by $\mathrm{C}_{\mathrm{DD}}$ whereas the load is powered from $\mathrm{C}_{\mathrm{ST}}$. Furthermore, this scheme, which will be referred to in this paper as two-way energy storage (TWS), allows the load to completely drain $\mathrm{C}_{\mathrm{ST}}$ without affecting the operation of the active power converter. In [16], [38], [39], $\mathrm{C}_{\mathrm{DD}}$ is charged initially through a passive path to start the active converter and, when the voltage on $\mathrm{C}_{\mathrm{ST}}$ is sufficient, the two capacitors are shorted or connected through a diode so that the incoming power sustains both the converter and the load. In the presented architecture, during the start-up phase $C_{D D}$ is passively charged trough a secondary passive rectifier, implemented with a NVC with a diode in series (NVCD), until the minimum voltage $V_{D D \min }$ required for properly powering the active conversion process. This phase is expected to be much shorter than in single storage systems because of the significantly lower value of $C_{D D}$ with respect to $C_{S T}$ for sustaining the power converter. Then, as soon as SECE is started, the energy flow is directed towards $C_{D D}$ until it is recharged at least to a higher voltage $V_{D D a c t \text { max }}$ in order to keep the converter supply in the required operating range, which is a priority task as it will be shown in the next paragraph. $V_{\text {DDact,max }}$ is not the maximum allowed supply voltage, but it is the higher threshold voltage of an hysteretic level comparator used for routing energy alternatively to $C_{S T}$ and $C_{D D}$ (i.e. for activation of $\mathrm{S}_{\mathrm{ST}}$ or $\mathrm{S}_{\mathrm{DD}}$ in Fig. 5). Then, the energy flow is diverted to $C_{S T}$ as long as $V_{D D}$ remains above a second threshold voltage $V_{\text {DDact,min }}$ chosen for preserving a high conversion efficiency. Below this voltage, energy is diverted to $C_{D D}$ again to keep the power converter functional. It holds that $V_{\text {DDmin }}<V_{\text {DDact,min }}<V_{\text {DDact,max }}$.

In order to assess the advantages of TWS, it is useful to compare the energy extracted by the NVCD passive interface, i.e. when the minimum baseline voltage is still not reached in a single storage system with a large capacitor, with the energy extracted by SECE. The output energy in passive operation (i.e. when SECE is not activated) $E_{P, B R}$ per half-wave can be evaluated by integrating the current through the diode in the passive path in Fig. 5 with a fixed output $V_{D D}$, assumed to be constant in the half-period (e.g. with a large capacitor or a supercapacitor) and the resulting expression for $E_{P, B R}$ is the following:

$E_{P, B R}=2 C_{P} V_{D D}\left(V_{P 0}-V_{D D}-V_{\gamma}\right)$
The SECE process has an intrinsic efficiency value $\eta_{S}$, defined as the energy transferred to the output divided by the energy removed from the transducer in a single charge extraction, which is also dependent on circuit parameters and components. In an ideal case it holds that $\eta_{S}=1$. In order to evaluate the performances of TWS, the effectiveness of SECE with NVC and $\mathrm{BR}$ are compared using the ratio $\eta_{P}=E_{P, B R} / E_{L}^{(N V C)}$. The ratio $\eta_{P}$ accounts for the efficiency of SECE with NVC and, approximating to unity the exponential term in $E_{L}{ }^{(N V C)}$, it can be written as:

$\eta_{P}=\frac{1}{\eta_{S}} \frac{V_{D D}}{V_{P 0}-V_{T}}\left(1-\frac{V_{D D}+V_{\gamma}}{V_{P 0}}\right)$.

The numerical evaluation of (6) is illustrated in Fig. 6 for some values of $\mathrm{V}_{\mathrm{P} 0}$ and it is clearly shown that the theoretical efficiency of the ideal SECE $\left(\eta_{S}=1\right)$ is higher than the BR in any case (as $\left.\eta_{\mathrm{P}}<1\right)$. This also holds with a non-ideal SECE with a sub-optimal efficiency $\eta_{S}=0.5$. Therefore, it is advisable to use SECE with respect to a passive rectifier in any configuration, as soon as it is possible. In addition, if a supercapacitor (from $\mathrm{mF}$ to the $\mathrm{F}$ range) is required, the use of a single energy storage element for supplying both the converter and the load is not optimal for the start-up phase, i.e. from $0 \mathrm{~V}$ to $V_{D D m i n}$, because this phase relies on a passive rectifier for energy harvesting. In the above considerations, RCI was not considered. Anyway, enabling also RCI reinforces the above conclusions, as it will be shown experimentally in Section V.

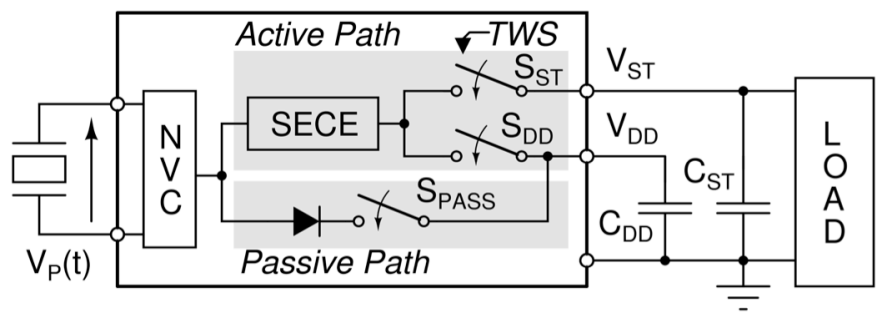

Fig. 5. Block diagram showing the active and passive charging paths in the converter and TWS for the active path.

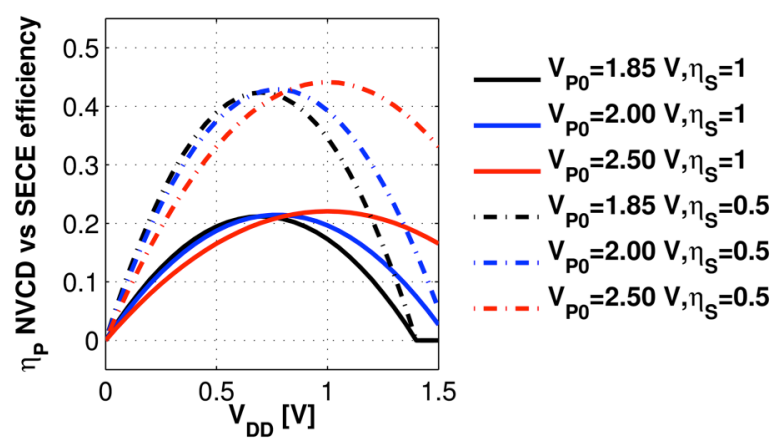

Fig. 6. Efficiency of passive charging of storage element (NVC+Diode) for start-up with respect to SECE. As can be pointed out, the efficiency is less than $50 \%$ even for SECE with a low conversion efficiency $\left(\eta_{S}=0.5\right)$. The values used for the evaluation are $V_{T} \cong 1 \mathrm{~V}$ (for generic MOSFETs) and $V_{\gamma}=0.35 \mathrm{~V}$ (for generic Schottky diodes). 


\section{CONVERTER ARCHITECTURE}

The architecture of the proposed converter implementing a self-starting SECE with RCI and TWS, which has been designed in a $0.32 \mu \mathrm{m}$ microelectronic technology from STMicroelectronics, is depicted in Fig. 7. The converter requires two external capacitors $C_{D D}$ and $C_{S T}$, two inductors $L_{I}$ and $L_{2}$ and a variable resistor $R_{R C I}$, which is used for setting the duration of RCI. In a future version of the converter, a single inductor can be utilized rather than $L_{1}$ and $L_{2}$ as the utilization factor of each inductor is very low. In fact, the use of a second inductor $L_{2}$ for the RCI circuit is not mandatory and only $L_{l}$ might be used for both the buck-boost converter and the RCI circuit, as the RCI phase can be executed immediately after a full cycle of the buck-boost converter. However, this would require major design changes in the control logic. Since the main purpose of this work was to assess the effectiveness of RCI, two separate inductors were used in this design for ease of implementation.

Once the converter has started active operations (i.e. SECE) the transducer is always kept in open circuit by the switch $\mathrm{S}_{\mathrm{S}}$, which is normally open. As the converter operates as a buckboost converter, there is never a direct conduction path from the transducer to $V_{S T}$ or $V_{D D}$. Moreover, RCI is performed directly on the transducer nodes and thus RCI is not affected by the load and by $V_{S T}$ or $V_{D D}$. RCI depends only on the rectifier characteristics, hence on the threshold voltage $V_{G S, t h}$ of the MOSFETs in the NVC, and on the series resistance of the associated $L_{2}-C_{P}$ circuit.

The converter draws nominally a quiescent current $I_{D D q}$ equal to $160 \mathrm{nA}$ when no energy extraction cycles are preformed (i.e. in idle state) at $V_{D D}=2.7 \mathrm{~V}$. The current drawn from each sub-circuit, obtained by simulations, is listed in Table I.

\section{A. Passive start-up}

This part of the circuit is shown in Fig. 8. The piezoelectric transducer is firstly connected to an NVC which outputs the rectified version $V_{R}$ of the input voltage $V_{P}$ (i.e. during the negative half-waves the sign is inverted). At start-up from a discharged state, a pMOS diode $\mathrm{M}_{\mathrm{PD}}$ and a depletion nMOS $\mathrm{M}_{\mathrm{Nd}}$ (i.e. a normally-closed switch) connect $V_{R}$ to $V_{D D}$ allowing the latter to be passively charged. The converter starts to operate actively (i.e. SECE is activated) as soon as $V_{D D} \geq V_{D D \min }=1.4 \mathrm{~V}$. At that voltage, an under-voltage lockout (UVLO1) circuit triggers and $\mathrm{M}_{\mathrm{Nd}}$ is turned off blocking the passive charging path towards $\mathrm{C}_{\mathrm{DD}}$ and then the buck-boost converter is activated and SECE is performed. An hysteresis of about $100 \mathrm{mV}$ is added to the UVLO in order to prevent undesired on-off switching due to noise and small variations on $V_{D D}$. Actually, the minimum required input voltage amplitude for a successful start-up operation is $V_{P O}=1.8 \mathrm{~V}$. However, as $C_{D D}$ is usually comparable with the output capacitance of the piezoelectric transducer (in this work $C_{D D} \cong 200-470 \mathrm{nF}$ ), it only takes a few oscillation periods in order to charge $C_{D D}$ up to $V_{D D \min }$ and start SECE. Once SECE and RCI are started, the converter can successfully extract energy with input voltages down to $0.7 \mathrm{~V}$.

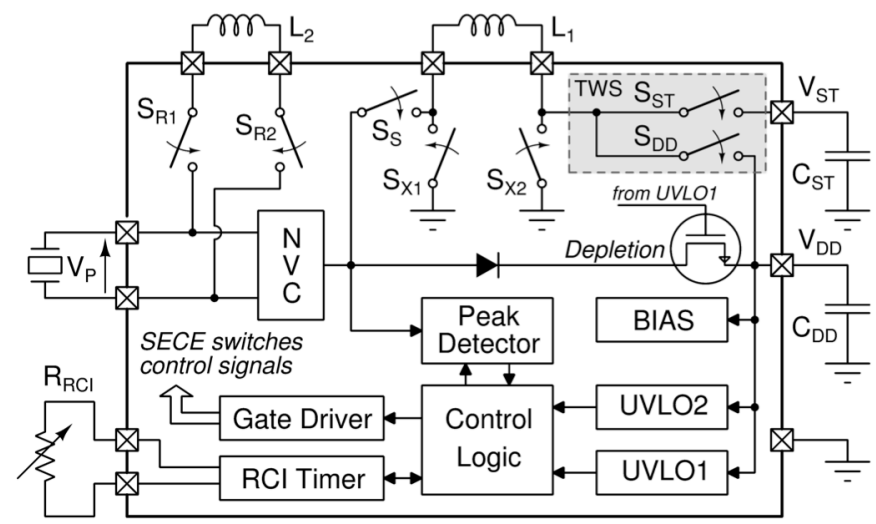

Fig. 7. Block diagram of the designed converter IC.

TABLE I SIMULATED QUIESCENT CURRENT DRAWN BY EACH SUB-CIRCUIT OF THE CONVERTER.

\begin{tabular}{lc}
\hline \hline Sub-circuit & Current [nA] \\
\hline Bias & 48 \\
\hline UVLO1 & 16 \\
\hline UVLO2 & 16 \\
\hline Peak Detector & 32 \\
\hline Bias (in Buck-boost converter) & 16 \\
\hline Other & 32 \\
\hline \hline
\end{tabular}

${ }^{1}$ This current is drawn by a comparator detecting whether $V_{S T}$ is greater than an externally applied voltage reference. Such function is not used by the converter but its consumption has been considered in the evaluation of the quiescent current as well as in the experimental results.

The passive start-up block draws $64 \mathrm{nA}$ nominally and embeds a supply-independent bias circuit which generates a reference current of $16 \mathrm{nA}$ and outputs the reference voltages $V_{B P}$ and $V_{B N}$ which are used as inputs for biasing all the analog circuitry of the IC.
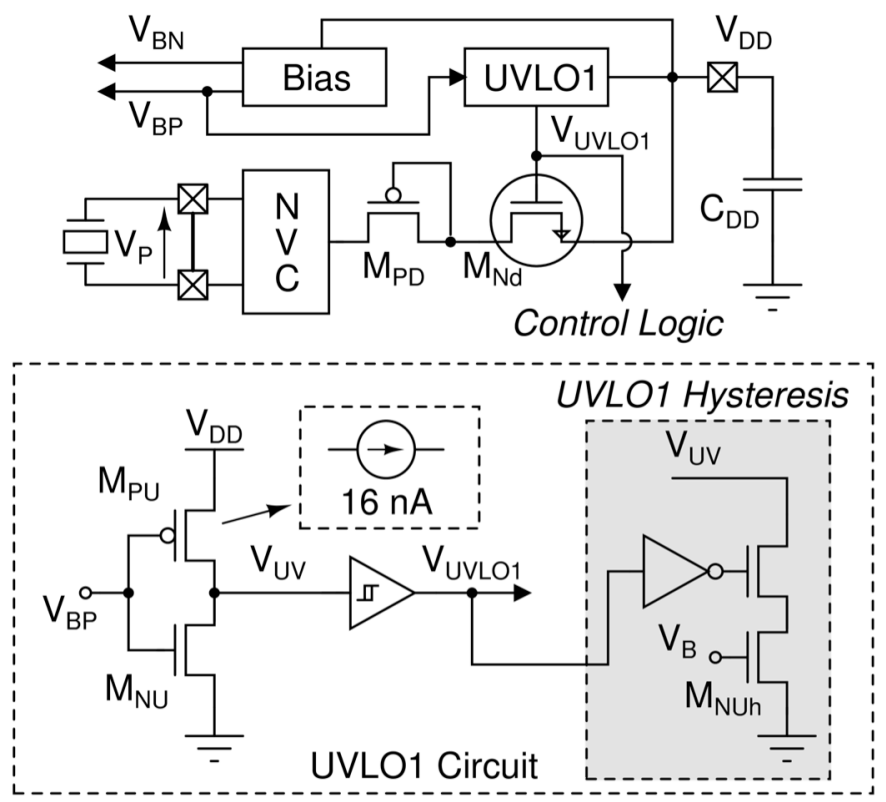

Fig. 8. Passive start-up circuit and details of UVLO1 circuit diagram. 


\section{B. Piezoelectric transducer interface}

The NVC output $V_{R}$ is tracked by an ultra-low power peak detector and an energy extraction cycle is performed on each maximum of $V_{R}$. The circuit diagram of the interface for the piezoelectric transducer is shown in Fig. 9. It is composed by an NVC for signal rectification, a switch for the connection to the main inductance $L_{l}$, a peak detector and the switches for RCI. The first stage of the peak detector is an input signal conditioning block and such stage is required in order to filter the input signals lower than the minimum value of $V_{P \min }=700 \mathrm{mV}$. It also includes an RC filter $\left(R_{f}\right.$ and $\left.C_{f}\right)$ with a $-3 \mathrm{~dB}$ cut-off frequency of $5.3 \mathrm{kHz}$ in order to smooth the spikes generated during RCI and prevent false peak detections. $\mathrm{M}_{\mathrm{F} 1}$ and $\mathrm{M}_{\mathrm{F} 3}$ are low threshold nMOS transistors. The next stage is composed of a voltage tracking circuit and a hysteretic comparator with hysteresis $V_{h}= \pm 15 \mathrm{mV}$. The static current drawn by the peak detector is as low as $32 \mathrm{nA}$, equally divided between the voltage tracking block $\left(\mathrm{OPA}_{\mathrm{PK}}\right)$ and the hysteretic comparator $\left(\mathrm{CMP}_{\mathrm{PK}}\right)$. The voltage tracking block charges $C_{\text {track }}$ with $\mathrm{M}_{\mathrm{P} 2}$ in order to keep $V_{\text {track }}=V_{R f}$. As charge on $C_{\text {track }}$ can only be added by $\mathrm{M}_{\mathrm{P} 2}$, a maximum is detected by the comparator when $V_{R f} \leq\left(V_{\text {track }}-V_{h}\right)$ and, in this case, the PEAK signal is set to $\mathrm{V}_{\mathrm{DD}}$. At the end of an energy extraction cycle $C_{\text {track }}$ is reset by $\mathrm{M}_{\mathrm{Nr}}$ in order to rightly track the next halfwave on $V_{R f}$ and detect the following maximum; $V_{R E S E T}$ is generated by the logic controller on the falling edge of CONV signal, which is activated by the control logic only during energy extractions. As $V_{P}$ (and thus $V_{R}$ ) can exceed $V_{D D}$, the amplifier $\mathrm{OPA}_{\mathrm{PK}}$, the current mirror $\mathrm{M}_{\mathrm{P} 1}-\mathrm{M}_{\mathrm{P} 2}$ and the comparator $\mathrm{CMP}_{\mathrm{PK}}$ are supplied by the highest voltage between $V_{D D}$ and $V_{R}$ by a dedicated bulk regulator circuit.

The switch connecting $V_{R}$ and $V_{L X I}$ is composed by both a nMOS and a pMOS and both gates are driven by a gate driver (GD) which, for the case of $\mathrm{M}_{\mathrm{PS}}$, is constantly supplied by the highest voltage between $V_{D D}, V_{L X I}$ and $V_{R}$ in order to completely turn off $\mathrm{M}_{\mathrm{PS}}$.

The RCI circuit is made of two nMOS switches $\mathrm{M}_{\mathrm{R} 1}$ and $\mathrm{M}_{\mathrm{R} 2}$, which connect $V_{P 1}$ and $V_{P 2}$ to the inductor $L_{2}$. The RCI phase is started by the logic controller as soon as the energy on $C_{P}$ has been extracted. In this implementation, the length of the RCI phase is set by the value of a resistor $\mathrm{R}_{\mathrm{RCI}}$, which alters the RC constant in a pulse generator (RCI Timer shown in Fig. 7). The timing of RCI is deeply analysed and illustrated in the next section, together with buck-boost converter operation.

\section{Buck-boost converter with TWS}

The buck-boost converter (circuit diagram shown in Fig. 10) is managed by a clock-less logic controller which implements an asynchronous finite state machine (FSM). When a maximum is detected PEAK is set to $V_{D D}$ and the FSM is activated from idle state. The end of each phase of the energy extraction process (energy transfer from $C_{P}$ to $L_{X}$ and from $L_{X}$ to $C_{S T}$ or $\left.C_{D D}\right)$ is dynamically determined by means of zero-voltage switching (ZVS) and zero-current switching (ZCS) detectors, This grants many degrees of freedom in the choice of the piezoelectric transducer, $C_{D D}, C_{S T}, L_{X}$, input power level and output voltage, because conversion timings are not hard-coded in the control circuits. While the converter is performing the second phase of the energy extraction process (i.e. energy transfer from $L_{X}$ to $C_{S T}$ or $C_{D D}$ ), the RCI is activated in order to invert the residual charge on $\mathrm{C}_{\mathrm{P}}$. The signals of the FSM, the input voltage $V_{P}$, and $V_{L X 2}$ are shown in Fig. 11. The extraction process starts as soon as the PEAK signal rises. The analog circuitry is normally turned off in idle state in order to minimize static current (down to $16 \mathrm{nA}$ ). When the extraction process starts, the dynamic bias block is turned on $(\mathrm{BM}=0$, active low) and, as it is the first phase of the energy extraction process, signals are set as follows: $V_{G X 2}=V_{D D}$ (which is the same signal as CONV in Fig. 9) and $V_{G X I}=0 \mathrm{~V}$. The end of the first phase is notified by the rising edge of ZVS signal. The second phase of the energy extraction is the transfer of energy from $L_{1}$ to $C_{S T}$ (or $C_{D D}$ ) with $V_{G X I}=V_{D D}$ and $V_{G X 2}=0 \mathrm{~V} ; V_{L X 2}$ is clamped to $V_{S T}$ in Fig. 11 which was forced, in this case, to $2 \mathrm{~V}$. Simultaneously, the RCI is performed on the piezoelectric transducer (RCI control signal high and voltage inversion on $V_{P}$ shown in the traces of Fig. 11). The second

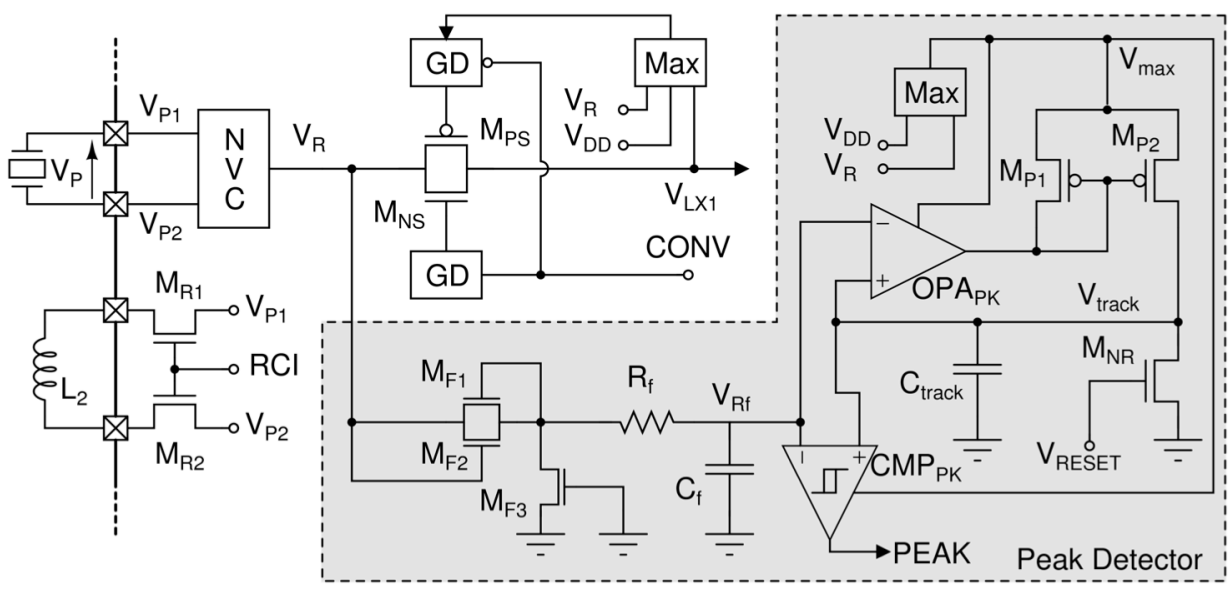

Fig. 9. Circuit diagram of the interface for the piezoelectric transducer. 


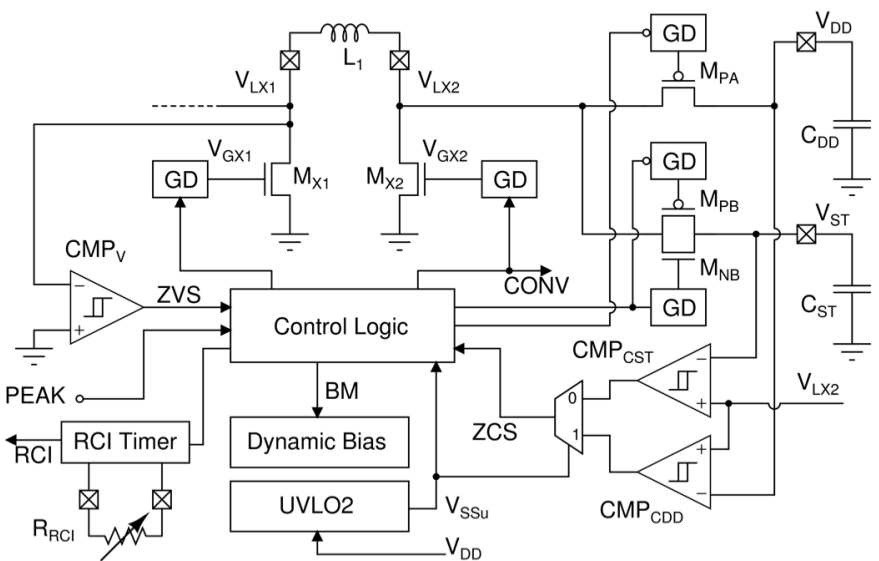

Fig. 10. Circuit diagram of the SECE converter.

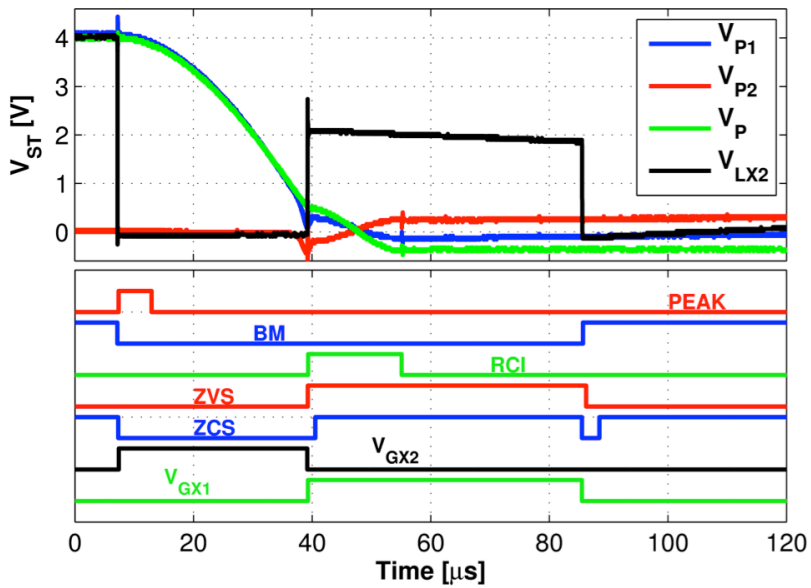

Fig. 11. Waveforms acquired from a sample device measurement during an energy extraction cycle. Both SECE and RCI are shown together with internal control signals (on bottom). In such acquisition, the energy was directed towards $C_{S T}$.

phase ends as all energy is moved to $C_{S T}$ (or $C_{D D}$ ) and this is notified by a falling edge of ZCS. The inputs of the FSM are normally masked and are enabled only during the FSM state in which they are relevant in order to prevent incorrect operations. ZCS is achieved by monitoring the voltage on $\mathrm{M}_{\mathrm{PA}}$ (or $\mathrm{M}_{\mathrm{PB}}$ ) due to its on-resistance and ZCS is effectively detected when $V_{S T}-V_{L X 2} \leq-15 \mathrm{mV}$ (or $V_{D D}-V_{L X 2} \leq-15 \mathrm{mV}$ ). Such scheme allows flexibility and adaptability at the cost of a lower efficiency for values of $V_{S T}$ approaching $5 \mathrm{~V}$ because $\mathrm{d} i_{L I} / \mathrm{d} t$ increases and the discharge time of $L_{l}$ becomes comparable with the propagation delay of the comparator $\mathrm{CMP}_{\mathrm{CST}}$ (or $\mathrm{CMP}_{\mathrm{CDD}}$ ) which is about $800 \mathrm{~ns}$.

The UVLO2 circuit in Fig. 10 is similar to UVLO1 in Fig. 8 but it has different thresholds which determine $V_{D D a c t, m i n}$ and $V_{\text {DDactmax }}$ UVLO2 switches it state when $V_{D D}$ rises above $2.6 \mathrm{~V}$ and when it drops below $2 \mathrm{~V}$. Its output $V_{S S u}$ notifies the control logic whether to charge $C_{S T}$ or to start a $C_{D D}$ charging sequence in order to provide energy to the converter itself. On the right of Fig. 12, TWS operation on a measurement with a sample of manufactured devices is illustrated and the $V_{S S u}$ signal, brought out of the chip with a test structure, is highlighted. The high state of the signal (about $1 \mathrm{~V}$ ) means that the UVLO2 circuit has detected that $V_{D D}$ is below the minimum value and thus the converter is forced to direct energy towards $C_{D D}$ instead of $C_{S T}$.

\section{EXPERIMENTAL RESULTS}

The power converter has been manufactured in a $0.32 \mu \mathrm{m}$ technology from STMicroelectronics in an active area of $0.95 \mathrm{~mm}^{2}$. The converter is placed in a $4.6 \mathrm{~mm}^{2}$ die, whose micrograph is shown on the left of Fig. 13. The setup used in the performed measurements is depicted on the right of Fig. 13 and the values of the used external components are shown in Table II. The chosen piezoelectric transducer is a Q220-A4303YB from Piezo Systems which has a nominal output capacitance $C_{P}=52 \mathrm{nF}$. Since the focus of the paper is on the converter design and the use of RCI and TWS, a first series of measurements has been performed with a real piezoelectric transducer in order to prove the functionalities of the proposed approach. Then, other experiments, whose aim was to

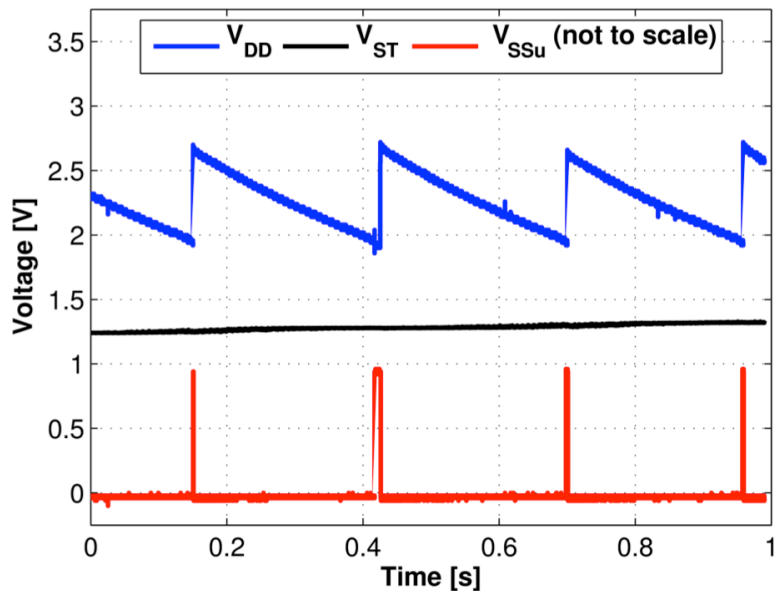

Fig. 12. (left) Waveforms acquired from a sample device measurement showing the TWS operating principle. $C_{S T}$ is slowly charged while $V_{D D}$ is charged only when its value is below a certain threshold, in order to keep the active converter enabled. The experimental conditions were: $V_{P O}=2 \mathrm{~V}, C_{P}=47.4 \mathrm{nF}$, $f=60 \mathrm{~Hz}, C_{S T}=66 \mu \mathrm{F}, C_{D D}=200 \mathrm{nF}, L_{1}=10 \mathrm{mH}, L_{2}=560 \mu \mathrm{H}$. (right) Detail of the TWS operations with the slow discharge of $V_{D D}$ due to self-consumption. $V_{S S u}$ (amplitude not to scale in the figure because it has been acquired from a test structure externally supplied) is the output of UVLO2 circuit and goes high when $C_{D D}$ needs a re-charge sequence. 
quantitatively characterize the performance of the circuit from an electric point of view, have been performed by emulating the transducer with laboratory equipment for higher accuracy and precision in the electrical quantities. The emulation was performed with an Agilent 33120A function generator setting the open-circuit voltage $V_{P 0}$ and a series-connected metallized polypropylene capacitor of $47.4 \mathrm{nF}$.

Firstly, as mentioned above, the IC has been tested with the piezoelectric transducer, as shown in Fig. 13. The load was emulated with a Keithley 2601 SMU forcing a load current of $8.25 \mu \mathrm{A}$ in order to obtain $V_{S T}=2.5 \mathrm{~V}$, which is a typical supply voltage for low power electronics. The piezoelectric transducer was excited with an electro-dynamic shaker vibrating at $\mathrm{f}_{\mathrm{P}}=50 \mathrm{~Hz}$ and with an acceleration $a_{R M S}=0.1 \mathrm{~g}$. With RCI enabled, the energy conversion efficiency, evaluated as the ratio between output power and available input energy times the energy extraction frequency (i.e. $f_{P} C_{P} V_{P \max }^{2}$ where $V_{P \max }$ is the transducer peak voltage generated by the nonlinear energy extraction process), has been experimentally determined at $72.2 \%$, with an extracted power from the transducer of $28.57 \mu \mathrm{W}$. However, we highlight that in the above experiment the IC was self-supplied with the harvested power and the reported efficiency value also includes the contribution of the IC intrinsic (ultra-low) power consumption. For this reason, the overall efficiency of the bare power conversion is higher than this value.

The second experiment is the measurement of the quiescent current of the IC with a Keithley 2601 SMU forcing $V_{D D}$ and measuring the drawn current while it is not performing any energy extraction and with an external bias on $V_{P}$ of $1 \mathrm{~V}$. The results are shown in Fig. 14. The IC draws about $160 \mathrm{nA}$ in its typical operating voltage ( $V_{D D}$ from $2 \mathrm{~V}$ to $3 \mathrm{~V}$ ) and this is very important for energy-limited scenarios. Then, by forcing $V_{D D}=2.7 \mathrm{~V}$ externally with the same SMU and filtering its output with an RC filter (with $R=1 \mathrm{M} \Omega, C=4.7 \mu \mathrm{F}$ ) the average current drawn from $V_{D D}$ by the converter has been measured, sweeping the input signal frequency from 20 to $100 \mathrm{~Hz}$ with $V_{P 0}=1.5 \mathrm{~V}$ and $C_{P}=47.4 \mathrm{nF}$. The obtained

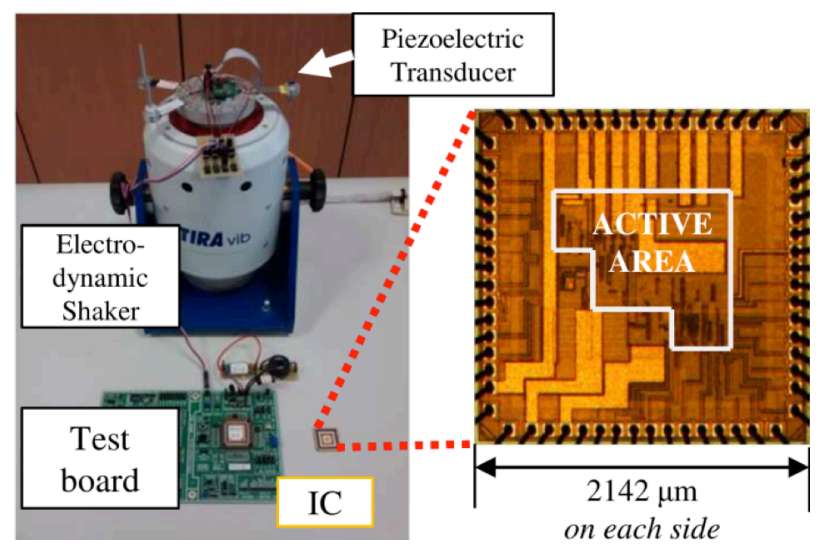

Fig. 13. Experimental setup used for measurements, based on the IC, a test board and a piezoelectric transducer stimulated by an electrodynamic shaker (left). Die micrograph with active area highlighted. (right).
TABLE II. VALUES OF EXTERNAL COMPONENT USED IN ALL EXPERIMENTS

\begin{tabular}{ccc}
\hline \hline Component & Value & DC Series Resistance \\
\hline$L_{1}$ & $10 \mathrm{mH}$ & $4.4 \Omega$ \\
$L_{2}$ & $560 \mu \mathrm{H}$ & $0.36 \Omega$ \\
$R_{R C I}$ & $1 \ldots .5 \mathrm{M} \Omega^{*}$ & - \\
$C_{P}$ & $47.4 \mathrm{nF}$ & - \\
$C_{D D}$ & $470 \mathrm{nF}$ & - \\
$C_{S T}$ & $10 \mathrm{mF}$ &.
\end{tabular}

* The value of $R_{R C I}$ must be tuned to set the duration of RCI to $\pi / \sqrt{L_{2} C_{P}}$ in order to obtain maximum RCI performance

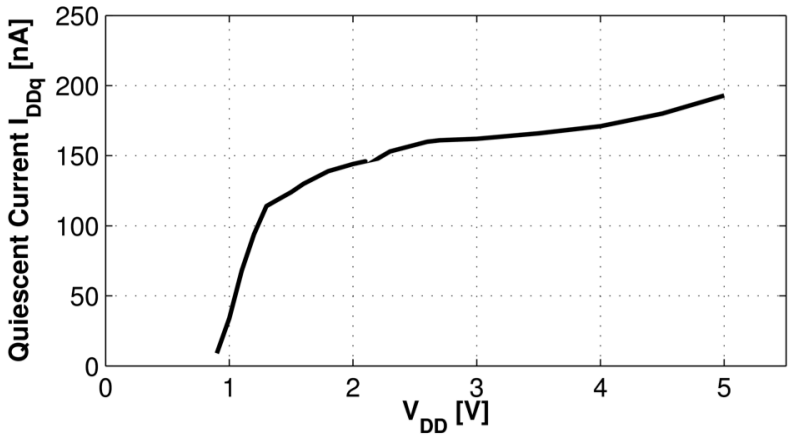

Fig. 14. Quiescent current drawn by the converter in stand-by state (no energy extractions are performed) for several $V_{D D}$ values.
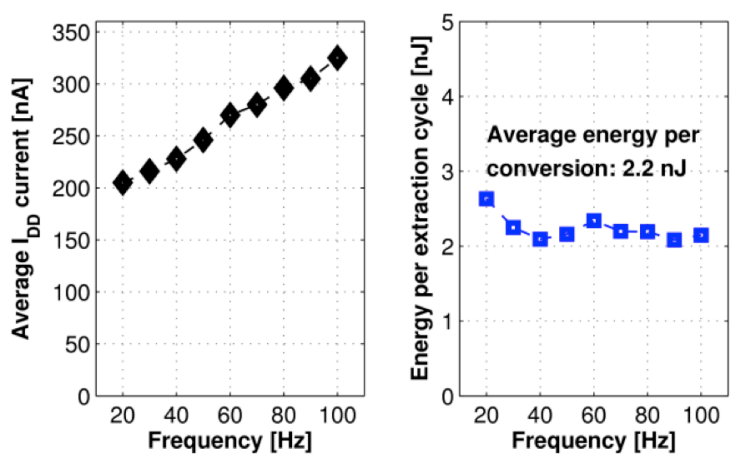

Fig. 15. (left) Measured average current $I_{D D}$ drawn on $V_{D D}$ for several input signal frequencies. (right). Energy required for a single energy extraction, calculated from the measured $I_{D D}$ when SECE-RCI is enabled.

results for the total current drawn $I_{D D}$ are show on the left of Fig. 15 whereas the dynamic energy required per energy extraction cycle is shown on the right of Fig. 15 and it is calculated from the difference between $I_{D D}$ and the measured $I_{D D q}$ of $166 \mathrm{nA}$ (at $V_{D D}=2.7 \mathrm{~V}$ ). The average value of the dynamic energy per conversion is $2.2 \mathrm{~nJ}$, and is small, typically less than $3 \%$, with respect to the available energy on a piezoelectric transducer $\left(0.5 C_{P} V_{P \max }{ }^{2}\right)$ with $C_{P}$ in the order of some tens of $\mathrm{nF}$.

In order to estimate the minimum input power required by the converter, a third experiment has been performed starting from an operating condition (with RCI enabled) and decreasing the input power down to $296 \mathrm{nW}\left(f_{P}=7 \mathrm{~Hz}\right.$ $V_{P 0}=500 \mathrm{mV}, C_{P}=47.4 \mathrm{nF}$ ). In such conditions the converter 
stops to provide energy to $C_{S T}$ but it is able to sustain itself and to continue performing SECE with RCI at $V_{D D}=1.54 \mathrm{~V}$.

The TWS has been validated through a fourth experiment: firstly, $V_{D D}$ and $\mathrm{V}_{\mathrm{ST}}$ have been connected together in order to emulate a classic single way storage (SWS) system; in a second time, the TWS technique has been enabled (i.e. $V_{S T}$ and $V_{D D}$ are independent). As previously discussed, in the start-up phase SECE and RCI can only be performed in the TWS system. The output voltage $\mathrm{V}_{\mathrm{ST}}$ has been acquired with a Tektronix MSO2024 digital oscilloscope in both cases and the results are shown in Fig. 16. TWS, together with SECE and RCI, is considerably improving the energy extraction process with an increase of more than 6.5 times of the harvested energy after $1000 \mathrm{~s}$ with respect to SWS (which, further, does not benefit from SECE and RCI) under the same input conditions (piezoelectric transducer emulated with $V_{P}=2 \mathrm{~V}$, $\left.f=60 \mathrm{~Hz}, C_{P}=47.4 \mathrm{nF}\right)$. The gain of TWS over SWS is present only when $V_{S T}<1.4 \mathrm{~V}$, because above this value SECE is activated also for SWS. However, this power management policy allows a quicker charging of the output from discharged states during start-up than with SWS, which becomes quite evident when $C_{S T}$ is a large capacitor, e.g. a supercapacitor in the $\mathrm{mF}$ range. In the latter case the start-up phase of a SWS can take up to several minutes or hours [30][37] with supercapacitors and weak and irregular vibrations. In this latter case, activating SECE in the initial phases significantly boost efficiency. In addition, the efficiency of SECE is quite independent from the output load condition [34][41], which results in an almost constant efficiency throughout the whole charging process.

In a fifth experiment, the efficiency of the energy transfer from $C_{P}$ to $C_{S T}$ has been measured and Fig. 17 shows the results for several values of $V_{P}$ spanning into the allowed range of operation of the IC. In this experiment $C_{S T}=66 \mu \mathrm{F}$ and a Keithley 2601 SMU was used as a constant current load. The measured peak efficiency is $85.3 \%$ for $V_{P}=2.35 \mathrm{~V}$. However, except for $V_{P 0}=1 \mathrm{~V}$, the efficiency is quite similar. The efficiency loss with $V_{P 0}=1 \mathrm{~V}$ is mainly due to incorrect ZCS timing: the time for energy transfer from $L_{I}$ to $C_{S T}$ is

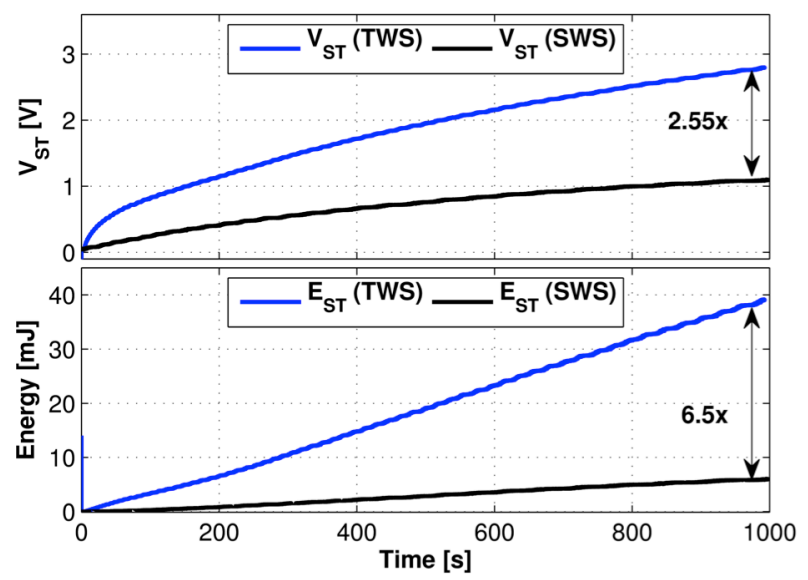

Fig. 16. Comparison of acquired $V_{S T}$ voltage with $C_{S T}=10 \mathrm{mF}$ in two configurations (SWS and TWS) with the same input excitation: piezoelectric transducer emulated with $V_{P 0}=2 \mathrm{~V}, f=60 \mathrm{~Hz}, C_{P}=47.4 \mathrm{nF}$.

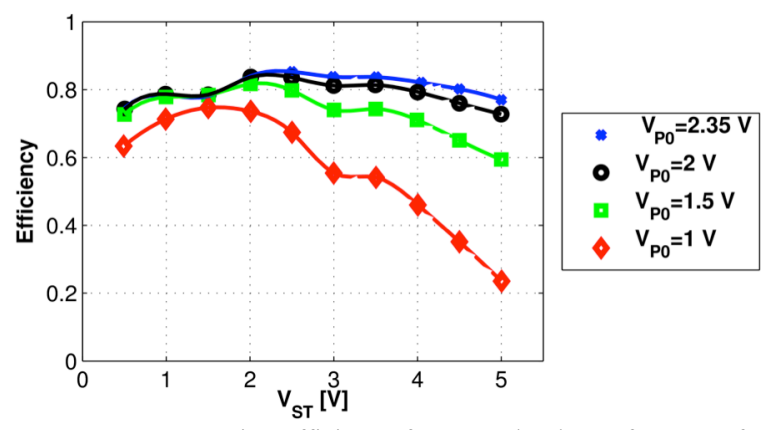

Fig. 17. Energy conversion efficiency for several values of $V_{P 0}$ at a frequency of $60 \mathrm{~Hz}$ with $V_{D D}=2.7 \mathrm{~V}$ externally supplied. Component values in Table II..

approximately proportional to the $V_{P} / V_{S T}$ ratio and, as charging progresses, it becomes comparable with the delay of the ZCS comparator and with the time required for detecting a negative (i.e. discharging $C_{S T}$ ) current on $L_{l}$. However, this is a necessary trade-off for reducing the intrinsic power consumption for operating with very low input power levels.

The output power dependence from the equivalent load resistor is shown in Fig. 18. Data are obtained from the fifth experiment. The equivalent load resistance is evaluated by dividing the voltage $V_{S T}$ by the current forced by SMU. The SMU current was chosen in order to obtain $V_{S T}$ ranging from $500 \mathrm{mV}$ (points on the left of Fig. 18) to $5 \mathrm{~V}$ (points on the right of Fig. 18), with steps of $500 \mathrm{mV}$. The dependency of output power from the load resistance is low, as expected from a SECE converter. In addition, two main effects can be observed. The first affects the output power (i.e. the energy transfer efficiency) for low value of load resistance and is caused by the increase of damping on the inductor $L_{l}$ current. This is due to the longer time needed for transferring the energy from $L_{1}$ to $C_{S T}$ and to the smaller $V_{G S}$ that turns on the $\mathrm{M}_{\mathrm{PB}}$ p-channel MOSFET in Fig. 10 (part of switch $\mathrm{S}_{\mathrm{ST}}$ in Fig. 7). On the other side, for higher load resistance (and thus $V_{S T}$ ) the main issue is due to the delay between the detection of the zero crossing of the inductor $L_{l}$ current and the turn-off of $\mathrm{S}_{\mathrm{ST}}$.

A sixth experiment has been used for the evaluation of the effectiveness of RCI and a comparison has been made with RCI enabled and disabled. After setting an operating point with $V_{S T}=1 \mathrm{~V}$ and then with $V_{S T}=2 \mathrm{~V}$, the output power of the converter has been measured with RCI enabled and, at a

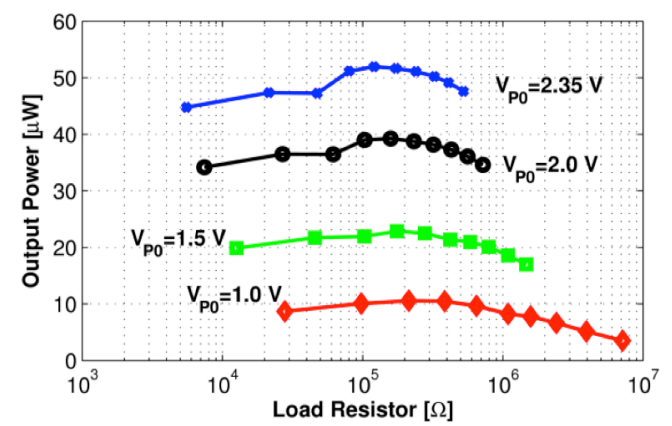

Fig. 18. Output power dependence on equivalent load resistance for different input voltage levels covering the allowed operating regions of the chip. 


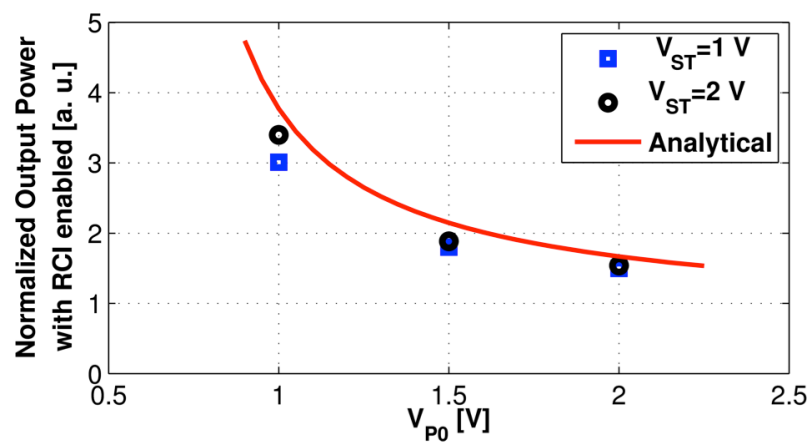

Fig. 19. Analytical performance improvement in output power of RCI and comparison with measured data on manufactured devices. For the analytical evaluation, $V_{T} \cong 1 \mathrm{~V}$ and the other component values are listed in Table II.

later stage, with RCI disabled and for different values of piezoelectric open-circuit voltage $V_{P O}$. The output power has been measured with the same methodology as the efficiency in the previous paragraph. The ratios between output power with and without RCI are illustrated in Fig. 19 in which the theoretic value, computed as the ratio of (4) over (2) is also shown. As it can be seen, the output power increases up to three times and there is a satisfactory agreement with analytical values (red line) and measured points. The mismatch in this case is mainly due to the resistive losses on the RCI switches, which do not allow to perform a full voltage inversion.

A comparison of the experimental results obtained on the designed converter with other recent works on SECE converters is shown in Table III.

\section{CONCLUSION}

A nano-power converter with an advanced energy storage power management policy and an input energy improvement scheme for piezoelectric energy harvesting has been presented. The IC draws a quiescent current as low as $160 \mathrm{nA}$ and about $500 \mathrm{nA}$ when actively extracting energy with an excitation of $60 \mathrm{~Hz}$. The measured peak efficiency is $85.3 \%$ for $V_{P 0}=2.35 \mathrm{~V}$. The RCI scheme improves the extracted power of more than $200 \%$ for $V_{P 0}=1 \mathrm{~V}$ and it is especially effective for weak vibrations. The proposed power management policy, the TWS, improves the overall converter performances during the start-up, significantly reducing the time required to charge an almost depleted supercapacitor. Moreover, TWS allows the load to drain all stored energy on $C_{S T}$ if required, and still prevents the converter from being stopped by the load current requirement, as the conveter supply is on a different capacitor than $C_{S T}$. In this prototype, two separate inductors have been used respectively for SECE and RCI. However, a single inductor may be time multiplexed for performing both function at the expenses of an increased circuit complexity. The size of the active area is $0.95 \mathrm{~mm}^{2}$ in a $0.32 \mu \mathrm{m}$ microelectronic technology, and the circuit may be used as a functional block in a $\mathrm{SoC}$ for energy autonomous WSN.
TABLE III. COMPARISON OF INTEGRATED SECE IMPLEMENTATION.

\begin{tabular}{cccc}
\hline Parameter & {$[41]$} & {$[16]$} & This Work \\
\hline Technology & $0.35 \mu \mathrm{m}$ & $\begin{array}{c}0.35 \mu \mathrm{m}+\text { off-chip } \\
\text { MOSFETs }\end{array}$ & $0.32 \mu \mathrm{m}$ \\
\hline Year & 2012 & 2013 & 2014 \\
\hline $\begin{array}{c}\text { Quiescent } \\
\text { current }\end{array}$ & $1.76 \mu \mathrm{A}^{1}$ & $330 \mathrm{nA}^{2}$ & $160 \mathrm{nA}$ \\
\hline $\begin{array}{c}\text { Maximum } \\
\text { input voltage }\end{array}$ & $20 \mathrm{~V}$ & $>70 \mathrm{~V}$ & $5 \mathrm{~V}$ \\
\hline $\begin{array}{c}\text { Maximum } \\
\text { output voltage }\end{array}$ & $5 \mathrm{~V}$ & $3.3 \mathrm{~V}$ & $5 \mathrm{~V}$ \\
\hline Features & PSCE & $\begin{array}{c}\text { MS-SECE, TWS } \\
\text { (off-chip) }\end{array}$ & RCI, TWS \\
\hline $\begin{array}{c}\text { Peak } \\
\text { Efficiency }\end{array}$ & $\begin{array}{c}85 \% \text { at } \\
V_{P}=12.8 \mathrm{~V}\end{array}$ & $\begin{array}{c}61 \% \text { at } \\
V_{P}=40 \mathrm{~V}\end{array}$ & $\begin{array}{c}85.3 \% \text { at } \\
V_{P}=4.6 \mathrm{~V}\end{array}$ \\
\hline \hline
\end{tabular}

${ }^{1}$ equivalent current estimated from reported power losses. ${ }^{2}$ equivalent current estimated from reported power consumptions.

\section{REFERENCES}

[1] S. Roundy and P. Wright, "A piezoelectric vibration based generator for wireless electronics," Smart Materials and Structures, vol. 13, no. 5, pp. 1131-1142, Oct. 2004.

[2] S. Mehraeen, S. Jagannathan, and K. Corzine, "Energy harvesting from vibration with alternate scavenging circuitry and tapered cantilever beam," IEEE Transactions on Industrial Electronics, vol. 57, no. 3, pp. 820-830, 2010.

[3] B. Yang and K.-S. Yun, "Piezoelectric shell structures as wearable energy harvesters for effective power generation at low-frequency movement," Sensors and Actuators A: Physical, Mar. 2012.

[4] J.-Q. Liu et al., "A MEMS-based piezoelectric power generator array for vibration energy harvesting," Microelectronics Journal, vol. 39, no. 5, pp. 802-806, May 2008.

[5] R. J. M. Vullers, R. V. Schaijk, I. Doms, C. Van Hoof, and R. Mertens, "Micropower energy harvesting," Solid State Electronics, vol. 53, no. 7, pp. 684-693, 2009.

[6] J. Iannacci, E. Serra, R. Di Criscienzo, G. Sordo, M. Gottardi, A. Borrielli, M. Bonaldi, T. Kuenzig, G. Schrag, G. Pandraud, and P. M. Sarro, "Multi-modal vibration based MEMS energy harvesters for ultralow power wireless functional nodes," Microsyst. Technol., vol. 20, no. 4-5, pp. 627-640, Dec. 2013.

[7] A. Bertacchini, S. Scorcioni, D. Dondi, L. Larcher, P. Pavan, M. T. Todaro, A. Campa, G. Caretto, S. Petroni, A. Passaseo, and M. De Vittorio, "AlN-based MEMS devices for vibrational energy harvesting applications," 2011 Proc. Eur. Solid-State Device Res. Conf., pp. 119122, Sep. 2011.

[8] S. Roundy, P. K. Wright, and J. Rabaey, "A study of low level vibrations as a power source for wireless sensor nodes," Computer Communications, vol. 26, no. 11, pp. 1131-1144, Jul. 2003

[9] G. K. Ottman, H. F. Hofmann, and G. Lesieutre, "Optimized piezoelectric energy harvesting circuit using step-down converter in discontinuous conduction mode," IEEE Transactions on Power Electronics, vol. 18, no. 2, pp. 696-703, Mar. 2003.

[10] G. K. Ottman, H. F. Hofmann, a. C. Bhatt, and G. a. Lesieutre, "Adaptive piezoelectric energy harvesting circuit for wireless remote power supply," IEEE Transactions on Power Electronics, vol. 17, no. 5, pp. 669-676, Sep. 2002.

[11] G. Szarka and B. Stark, "Review of Power Conditioning for Kinetic Energy Harvesting Systems," IEEE Transactions on Power Electronics, vol. 27, no. 2, pp. 803-815, 2012.

[12] E. Lefeuvre, A. Badel, C. Richard, and D. Guyomar, "Piezoelectric energy harvesting device optimization by synchronous electric charge extraction,” J. Intell. Mat. Syst. Str., vol. 16, pp. 865-876, Oct. 2005.

[13] E. Lefeuvre, A. Badel, C. Richard, L. Petit, and D. Guyomar, "A comparison between several vibration-powered piezoelectric generators for standalone systems," Sensors and Actuators A: Physical, vol. 126, no. 2, pp. 405-416, Feb. 2006 
[14] T. Paing and R. Zane, "Design and optimization of an adaptive nonlinear piezoelectric energy harvester," in 2011 Twenty-Sixth Annual IEEE Applied Power Electronics Conference and Exposition (APEC), 2011, pp. 412-418.

[15] Y. Wu, A. Badel, F. Formosa, W. Liu, A.E. Agbossou, "Piezoelectric vibration energy harvesting by optimized synchronous electric charge extraction," J. Intell. Mater. Syst. Struct., 24 (12) (2012), pp. 1445-1458

[16] Gasnier, P.; Willemin, J.; Boisseau, S.; Despesse, G.; Condemine, C.; Gouvernet, G.; Chaillout, J.-J., "An Autonomous Piezoelectric Energy Harvesting IC Based on a Synchronous Multi-Shot Technique," SolidState Circuits, IEEE Journal of, vol.49, no.7, pp.1561,1570, July 2014

[17] Guyomar, D.; Badel, A.; Lefeuvre, E.; Richard, C., "Toward energy harvesting using active materials and conversion improvement by nonlinear processing," Ultrasonics, Ferroelectrics, and Frequency Control, IEEE Transactions on, vol.52, no.4, pp.584,595, April 2005

[18] M. Lallart, L. Garbuio, L. Petit, C. Richard, and D. Guyomar, "Double synchronized switch harvesting (DSSH): a new energy harvesting scheme for efficient energy extraction.," IEEE Transactions on Ultrasonics, Ferroelectrics, and Frequency Control, vol. 55, no. 10, pp. 2119-30, Oct. 2008.

[19] L. Garbuio, M. Lallart, D. Guyomar, C. Richard, and D. Audigier, "Mechanical energy harvester with ultralow threshold rectification based on SSHI nonlinear technique," IEEE Transactions on Industrial Electronics, vol. 56, no. 4, pp. 1048-1056, 2009.

[20] Y. Ramadass and A. Chandrakasan, "An Efficient Piezoelectric Energy Harvesting Interface Circuit Using a Bias-Flip Rectifier and Shared Inductor," IEEE Journal of Solid-State Circuits, vol. 45, no. 1, pp. 189204, 2010.

[21] N. Krihely and S. Ben-Yaakov, "Self-Contained Resonant Rectifier for Piezoelectric Sources Under Variable Mechanical Excitation," IEEE Transactions on Power Electronics, vol. 26, no. 2, pp. 612-621, Feb. 2011

[22] C. Peters, J. Handwerker, D. Maurath, and Y. Manoli, "An Ultra-LowVoltage Active Rectifier for Energy Harvesting Applications," Circuits and Systems (ISCAS), Proceedings of 2010 IEEE, pp. 889-892, 2010.

[23] A. Romani et al., "Dynamic switching conversion for piezoelectric energy harvesting systems," 2008 IEEE Sensors, pp. 689-692, Oct. 2008.

[24] Y. Sun, N. H. Hieu, C.-J. Jeong, and S.-G. Lee, “An Integrated HighPerformance Active Rectifier for Piezoelectric Vibration Energy Harvesting Systems," IEEE Transactions on Power Electronics, vol. 27, no. 2, pp. 623-627, Feb. 2012.

[25] M. Dini, M. Filippi, A. Romani, V. Bottarel, G. Ricotti. M. Tartagni, "A nano-power energy harvesting IC for arrays of piezoelectric transducers," Proc. SPIE 8763, Smart Sensors, Actuators, and MEMS VI, 87631O, May 17, 2013, doi:10.1117/12.2017406.

[26] Romani, A; Paganelli, R.P.; Sangiorgi, E.; Tartagni, M., "Joint Modeling of Piezoelectric Transducers and Power Conversion Circuits for Energy Harvesting Applications," Sensors Journal, IEEE , vol.13, no.3, pp.916,925, March 2013 doi: 10.1109/JSEN.2012.2219580

[27] F. I. Simjee and P. H. Chou, "Efficient Charging of Supercapacitors for Extended Lifetime of Wireless Sensor Nodes," IEEE Transactions on Power Electronics, vol. 23, no. 3, pp. 1526-1536, May 2008.

[28] D. Pech et al., "Ultrahigh-power micrometre-sized supercapacitors based on onion-like carbon.," Nature nanotechnology, vol. 5, no. 9, pp. 651-4, Sep. 2010.

[29] P. Mars and D. McIntosh, "Using a Supercapacitor to Power Wireless Nodes from a Low Power Source such as a 3V Button Battery," in 2009 Sixth International Conference on Information Technology: New Generations, 2009, pp. 69-78.

[30] Aktakka, E.E.; Najafi, K., "A Micro Inertial Energy Harvesting Platform With Self-Supplied Power Management Circuit for Autonomous Wireless Sensor Nodes," Solid-State Circuits, IEEE Journal of , vol.49, no.9, pp.2017,2029, Sept. 2014 doi: 10.1109/JSSC.2014.2331953

[31] Dongwon Kwon; Rincon-Mora, G.A, "A single-inductor $0.35 \mu \mathrm{m}$ CMOS energy-investing piezoelectric harvester," Solid-State Circuits Conference Digest of Technical Papers (ISSCC), 2013 IEEE International , vol., no., pp.78,79, 17-21 Feb. 2013 doi: 10.1109/ISSCC.2013.6487645

[32] D. Maurath, P. F. Becker, D. Spreemann, and Y. Manoli, "Efficient energy harvesting with electromagnetic energy transducers using active low-voltage rectification and maximum power point tracking," SolidState Circuits, IEEE J., vol. 47, no. 6, pp. 1369-1380, 2012

[33] C. van Liempd, S. Stanzione, Y. Allasasmeh, and C. Van Hoof, "A $1 \mu \mathrm{W}$-to-1mW Energy-Aware Interface IC for Piezoelectric Harvesting with 40nA Quiescent Current and Zero-Bias Active Rectifiers," SolidState Circuits Conf. Dig. Tech. Pap. (ISSCC), 2013 IEEE Int., pp. 7678, 2013

[34] A. Romani, M. Filippi, M. Tartagni, "Micro-power Design of a Fully Autonomous Energy Harvesting Circuit for Arrays of Piezoelectric Transducers", IEEE Transactions on Power Electronics, vol. 29, no. 2, pp. 729-739, 2014

[35] Dicken, J., Mitcheson, P. D., Stoianov, I., \& Yeatman, E. M. (2012). Power-Extraction Circuits for Piezoelectric Energy Harvesters in Miniature and Low-Power Applications. Power Electronics, IEEE Transactions on, 27(11), 4514-4529. doi:10.1109/TPEL.2012.2192291

[36] Yang Sun; Nguyen Huy Hieu; Chang-Jin Jeong; Sang-Gug Lee, "An Integrated High-Performance Active Rectifier for Piezoelectric Vibration Energy Harvesting Systems," Power Electronics, IEEE Transactions on , vol.27, no.2, pp.623,627, Feb. 2012

[37] E. S. Leland, E. M. Lai, and P. K. Wright, "A Self-Powered Wireless Sensor for Indoor Environmental Monitoring," in Proc. of the Wireless Networking Symposium (WNCG), Austin, TX, USA, October 20-22 2004.

[38] Gasnier, P.; Willemin, J.; Chaillout, J.-J.; Condemine, C.; Despesse, G.; Boisseau, S.; Gouvernet, G.; Barla, C., "Power conversion and integrated circuit architecture for high voltage piezoelectric energy harvesting," New Circuits and Systems Conference (NEWCAS), 2012 IEEE 10th International, vol., no., pp.377,380, 17-20 June 2012

[39] Boisseau et al., "Self-starting power management circuits for piezoelectric and electret-based electrostatic mechanical energy harvesters", POWERMEMS, 2013

[40] Dini, M.; Filippi, M.; Tartagni, M.; Romani, A., "A nano-power power management IC for piezoelectric energy harvesting applications," Ph.D. Research in Microelectronics and Electronics (PRIME), 2013 9th Conference on , vol., no., pp.269,272, 24-27 June 2013

[41] T. Hehn, F. Hagedorn, D. Maurath, D. Marinkovic, I. Kuehne, A. Frey, Y. Manoli, "A Fully Autonomous Integrated Interface Circuit for Piezoelectric Harvesters," Solid-State Circuits, IEEE J., vol. 47, no. 9, pp. 2185-2198, 2012. 


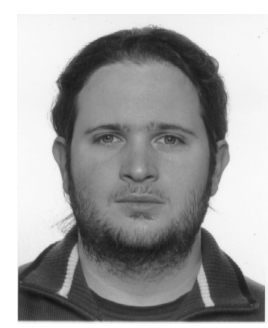

Michele Dini received the B.S. and M.S. degrees in electrical engineering from the University of Bologna, Cesena, Italy, in 2008 and 2011 respectively. Since 2012, he has been working towards his Ph.D. degree in electrical engineering at the Advanced Research Center on Electronic Systems and at the Department of Electrical, Electronic, and Information Engineering of the same university. He has been involved with energy harvesting systems, design of CMOS power converters and power management circuits.

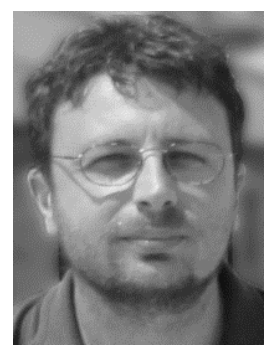

Aldo Romani received the Dr.Eng. degree in electrical engineering and Ph.D. degree in electrical engineering, computer science, and telecommunications from the University of Bologna, Bologna, Italy, in 2001 and 2005 , respectively. In 2008, he joined the University of Bologna, Cesena, Italy, as an Assistant Professor. He has authored or coauthored more than 40 international scientific publications. He has been involved with CMOS integrated sensors, applications of piezoelectric materials, and energy harvesting systems. Dr. Romani was a co-recipient of the 2004 Jan Van Vessem Award of the IEEE International Solid-State Circuits Conference.

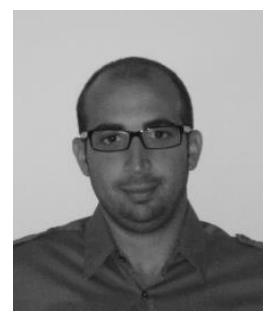

Matteo Filippi received the degree in electrical engineering in 2011 from the University of Bologna, Cesena, Italy. Since 2012, he has been with the Advanced Research Center on Electronic Systems and with the Department of Electrical, Electronic, and Information Engineering, University of Bologna, Cesena, Italy, where he has been involved in energy harvesting systems, micropower electronics, and CMOS design.

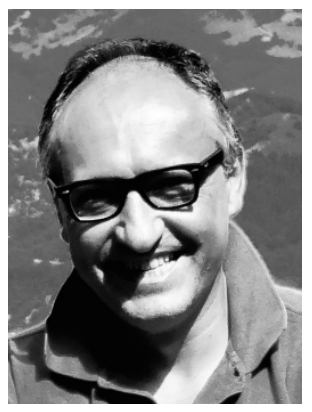

Marco Tartagni (M'98) received the M.S. degree in electrical engineering in 1988 and the Ph.D. degree in electrical engineering and computer Sciences in 1993 both from the University of Bologna, Bologna, Italy. In 1994, he joined the Department of Electrical Engineering, California Institute of Technology, Pasadena, CA, USA, as a Research Fellow. Since March 1995, he has been with the Department of Electronics, University of Bologna, where he is currently an Associate Professor. In 1997, he designed the first silicon-only fingerprint capacitive sensor. In 1999, he co-founded the company Silicon Biosystems active in the field of lab-on-a-chip devices. From 2005 to 2008, he was an European coordinator of FP6 Receptronics in the Nanotechnology thematic area. Since 2008, he has been a coordinator of the Working Group on Energy Autonomous Systems within the European CATRENE initiative. Since 2014, he has been a Member of the Scientific Board and Team Leader of the joint STMicroelectronics and Bologna University lab for sensor design. He has coauthored more than 100 refereed scientific publications, 18 granted U.S. patents, and 11 European and WIPO patents. Prof. Tartagni was a co-recipient of the 2004 Jan Van Vessem Award of the IEEE International Solid-State Circuits Conference. 